\title{
ON THE FINITE SIMILARITY GROUPS
}

\author{
PETER LORIMER
}

(Received 22 February 1968)

A result contained in a previous paper [1] of the author is

Theorem 1. If $(G, K, H)$ is a $T_{3}$-triple, $G$ is finite, $a$ is an involution contained in $G-N(K)$ and $H \cap K^{a}=1$, then the factor group of $G$ over its centre is isomorphic to a group of similarities over a finite field.

Here we refine this result as follows

THEOREM 2. Under the hypotheses of Theorem 1, G is the direct product of an abelian group and a group isomorphic to a group of similarities over a finite field.

The converse of this theorem is also true as will be pointed out in section 4 .

The concept of a $T_{3}$-triple is defined in the next section.

\section{Notations and Definitions}

$H \triangleleft G$ means that $H$ is a normal subgroup of the group $G$; $N(H)$ is the normalizer of $H ; C(h)$ is the centralizer of $h ;|H|$ is the order of $H$; $(G ; H)$ is the index of $H$ in $G$; $a^{x}=x^{-1} a x ; H^{x}=x^{-1} H x$; if $H \triangleleft G$, then $G / H$ is the factor group of $H$ in $G ; Z(G)$ is the centre of $G ; G-H$ is the set of elements contained in $G$ but not in $H ; A \times B$ is the direct product of the groups $A$ and $B$; if $F$ is a field, then $S(2, F)$ is the group of similarity mappings $z \rightarrow a z+b, a \neq 0$ over the field $F$.

Definition. If $K$ and $H$ are subgroups of a group $G, H \triangleleft K$ and whenever $a$ and $a^{b}$ are members of $G-K$ there exists exactly one $h \in H$ with the property $a^{h}=a^{b}$, then $(G, K, H)$ is called a $T_{3}$-triple and $G$ is called a $T_{3}$-group.

\section{Previous results}

In order to avoid repetition we shall use some of the results of [1] without proof. The most important of these is Theorem 1 stated at the beginning of this paper but in addition we have 
Proposition 2.1. $Z(G)=K \cap K^{a}$.

Proposition 2.2. With $F$ as defined in Theorem $1, H$ is isomorphic to the group of mappings $z \rightarrow a z, a \neq 0$, over $F$.

Proposition 2.3. $K=H \times Z(G)$.

Proposition 2.4. $G$ is doubly transitive on the cosets of $K$ in $G$.

Proposition 2.5. $N(K)=K$.

Proposition 2.6. $|G|=|K|(|H|+1)$.

All these propositions hold under the assumption of the hypotheses of Theorem 1. In addition we will use the properties of the similarity groups where necessary.

\section{The proof of Theorem 2}

Throughout this section we will assume that $(G, K, H)$ is a $T_{3}$-triple satisfying the hypotheses of Theorem 1 . We will denote the order of $H$ by $n-1$ and the order of $Z(G)$ by $m$. From propositions 2.3 and 2.6 it then follows that $|G|=n|K|=m n(n-1)$.

LEMMA 3.1. $K$ is abelian.

Proof. By propositions 2.2 and $2.3 K$ is the direct product of two abelian groups and is hence abelian.

LEMma 3.2. If $g \in G$ then either

(1) $g \in Z(G)$ or

(2) g has exactly one conjugate in $K$ and has $n$ conjugates in $G$, or

(3) $g$ has no conjugate in $K$ and has $n-1$ conjugates in $G$.

Proof. We will show first that no element of $G$ has two conjugates in $K$.

Suppose $g^{x} \in K, g^{y} \in K, g^{x} \neq g^{y}$. Then $g \in K^{x^{-1}} \cap K^{y^{-1}}$. If $K^{x^{-1}} \neq K^{y^{-1}}$, then, by Proposition 2.4, $g$ has a conjugate in $K \cap K^{a}$ which is the centre of $G$ (Proposition 2.1). Hence $g$ is contained in the centre of $G$ which is a contradiction.

Alternatively $K^{x^{-1}}=K^{v^{-1}}$ so that $x^{-1} y \in N(K)=K$ by Proposition 2.5. Now $g^{x} \in K$ so that by Lemma 3.1 $\left(g^{x}\right)^{x^{-1} y}=g^{x}$. Hence $g^{x}=g^{y}$ which is a contradiction.

Hence no element of $G$ has two conjugates in $K$.

Now, by the property $T_{3}$, if an element of $G$ has a conjugate outside $K$ it has exactly $|H|=n-1$ conjugates outside $K$. This proves Lemma 3.2.

By Theorem $1, G / Z(G)$ is isomorphic to a group $S(2, F)$. The group of 
translations $z \rightarrow z+b$ is a normal subgroup of this group so that $G$ has a subgroup $D$ which contains $Z(G)$ and has the property that $D / Z(G)$ is isomorphic to this group of translations. Moreover $D / Z(G) \triangleleft G / Z(G)$ so that $D \triangleleft G$.

Leмma 3.3. $D$ consists of the centre of $G$ together with all the elements of $G$ which leave no coset of $K$ fixed.

Proof. We note first that $D / Z(G)$ consists of the elements of $G / Z(G)$ which leave no coset of $K \mid Z(G)$ fixed, together with the identity $Z(G)$ of $G / Z(G)$.

If $g \in G$ and $g$ fixes a coset of $K$ in $G$ then the $\operatorname{coset} g Z(G)$ fixes a coset of $K / Z(G)$ in $G / Z(G)$. Conversely if $g$ fixes no coset of $K$ in $G$, then $g Z(G)$ fixes no coset of $K / Z(G)$ in $G / Z(G)$.

\section{Lemma 3.4. $D$ is abelian.}

Proof. Suppose $g \in D-Z(G)$. Then by Lemma $3.2 \mathrm{~g}$ has $n-1$ conjugates in $G$. But $G$ has order $m n(n-1)$ so that $C(g)$ has order $m n$. Now $Z(G) \subseteq C(g)$ so that $C(g) / Z(G)$ is defined and is a subgroup of $G / Z(G)$ of order $n$. But the only subgroup of $G / Z(G)$ having order $n$ is $D / Z(G)$ so that $C(g) / Z(G)=D / Z(G)$ and so $C(g)=D$.

Hence $D-Z(G) \subseteq Z(D)$ and clearly $Z(G) \subseteq Z(D)$ which proves the lemma.

Lemma 3.5. Let $\sigma$ be a fixed element of $H, \sigma \neq 1$, and let $E$ be the set of elements of $G$ of the form $x^{-1} x^{\sigma}, x \in D$. Then $E$ is a complement of $Z(G)$ in $D$, i.e. $D=Z(G) \times E$.

Proof. $D \triangleleft G$ so that if $x \in D$, also $x^{\sigma} \in D$. Hence $E \subseteq D$.

If $x^{-1} x^{\sigma} \in D$ and $y^{-1} y^{\sigma} \in D$, then $\left(x^{-1} x^{\sigma}\right)\left(y^{-1} y^{\sigma}\right)^{-1}=\left(x y^{-1}\right)^{-1}\left(x y^{-1}\right)^{\sigma}$ since $D$ is abelian. Hence $\left(x^{-1} x^{\sigma}\right)\left(y^{-1} y^{\sigma}\right)^{-1} \in E$ so that $E$ is a subgroup of $D$.

If $x^{-1} x^{\sigma} \in Z(G)$ then $x^{\sigma} \in x Z(G)$. Hence $\sigma Z(G)$ commutes with $x Z(G)$ in $G / Z(G)$. Now $\sigma Z(G)$ is not a translation and hence commutes with no translation of $G / Z(G)$. Hence $x Z(G)=Z(G)$ i.e. $x \in Z(G)$. Thus $x^{\sigma}=x$ so that $x^{-1} x^{\sigma}=1$. Hence $Z(G) \cap E=1$.

Suppose $g \in D$. Then $g Z(G) \in D / Z(G)$ which is the group of translations of $G / Z(G)$. Hence there exists $x \in D$ such that

$$
(x Z(G))^{-1}(x Z(G))^{\sigma Z(G)}=g Z(G) .
$$

Then $x^{-1} x^{\sigma} \in g Z(G)$ so that $D=Z(G) E$.

Clearly $E \triangleleft D(D$ abelian) so that $D=Z(G) \times E$.

LEMMA 3.6. $E \triangleleft G$. 
Proof. Suppose $x^{-1} x^{\sigma} \in E, x \in D, x^{-1} x^{\sigma} \neq 1$ and suppose $g \in G$. By Lemma 3.5, $x^{-1} x^{\sigma} \in D-Z(G)$ so that by Lemma 3.3, $x^{-1} x^{\sigma}$ has no conjugate in $K$. Hence, by the property $T_{3}$ there exists $h \in H$ such that $\left(x^{-1} x^{\sigma}\right)^{h}=\left(x^{-1} x^{\sigma}\right)^{g}$. But $H$ is abelian so that $\left(x^{-1} x^{\sigma}\right)^{h}=\left(x^{h}\right)^{-1}\left(x^{h}\right)^{\sigma} \in E$. Thus $\left(x^{-1} x^{\sigma}\right)^{g} \in E$ so that $E \triangleleft G$.

LEMMA 3.7. $H E$ is a normal subgroup of $G$ and is a complement of $Z(G)$ in $G$ i.e. $G=Z(G) \times H E$.

Proor. $E \triangleleft G$ and $H$ is a subgroup of $G$ so that $H E$ is a subgroup of $G$.

To prove that $H E$ is a normal subgroup of $G$ it will be sufficient to show that $H E$ contains every conjugate of every element of $H$.

Suppose $h \in H, h \neq 1$. Then by Lemma 3.2, $h$ has $n$ conjugates in $G$. If $x, y \in E, x \neq y$ and $h^{x}=h^{y}$, then $h^{x y^{-1}}=h$, so that $\left(x y^{-1}\right)^{h}=x y^{-1}$. Now, from Lemmas 3.3 and 3.5, $x y^{-1}$ has no conjugate in $K$ so that by the property $T_{3}$ there is a unique $h_{1} \in H$ with the property $\left(x y^{-1}\right)^{h_{1}}=x y^{-1}$, namely $h_{1}=1$. Hence $h=1$ which is a contradiction. Hence conjugates of $h$ by different elements of $E$ are different.

Now $|D|=m n$ and $|Z(G)|=m$ so that by Lemma 3.5, $|E|=n$. Hence if $h \in H, h \neq 1, h$ has $n$ conjugates in $H E$ by elements of $E$ and by the above this is the complete set of conjugates of $h$ in $G$. Hence $H E \triangleleft G$.

Clearly $H E \cap Z(G)=1$ and $|H E|=n(n-1),|Z(G)|=m$ so that $Z(G) H E=G$.

Hence we have proved that $G=Z(G) \times H E$.

This is sufficient to prove Theorem 2 .

\section{The converse of Theorem 2}

It remains now to prove

THEOREM 3. Let $F$ be a field and $H$ the subgroup of $S(2, F)$ consisting of similarities of the form $z \rightarrow a z, a \neq 0$. If $A$ is an abelian group then $(A \times S(2, F), A \times H, H)$ is a $T_{3}$-triple and moreover satisfies the other hypotheses of Theorem 1.

Proof. We prove only that $(A \times S(2, F), A \times H, H)$ is a $T_{3}$-triple.

$S(2, F)$ may be represented as the group of matrices of the form $\left(\begin{array}{ll}a & b \\ 0 & 1\end{array}\right), a, b \in F, a \neq 0$ in which case $H$ may be taken as the subgroup of matrices of the form $\left(\begin{array}{ll}a & 0 \\ 0 & 1\end{array}\right), a \in F, a \neq 0 . A \times S(2, F)$ may be then taken as the set of pairs of the form $\left(\alpha,\left(\begin{array}{ll}a & b \\ 0 & 1\end{array}\right)\right)$ with $\alpha \in A, a, b \in F, a \neq 0$ and multiplication defined elementwise.

A necessary condition that $\left(\alpha,\left(\begin{array}{ll}a & b \\ 0 & 1\end{array}\right)\right)$ and $\left(\beta,\left(\begin{array}{ll}c & d \\ 0 & 1\end{array}\right)\right)$ be conjugate is easily seen to be $\alpha=\beta$ and $a=c$. 
Suppose $\left(\alpha,\left(\begin{array}{ll}a & b \\ 0 & 1\end{array}\right)\right),\left(\alpha,\left(\begin{array}{ll}a & d \\ 0 & 1\end{array}\right)\right)$ are conjugate and neither are contained in $A \times H$. Then $b \neq 0, d \neq 0$. We must show that the equation

$$
\left(1,\left(\begin{array}{ll}
x & 0 \\
0 & 1
\end{array}\right)\right)\left(\alpha,\left(\begin{array}{ll}
a & b \\
0 & 1
\end{array}\right)\right)=\left(\alpha,\left(\begin{array}{ll}
a & d \\
0 & 1
\end{array}\right)\right)\left(1,\left(\begin{array}{ll}
x & 0 \\
0 & 1
\end{array}\right)\right)
$$

has a unique solution for $x$. This is easily seen to be $x=b^{-1} d$.

\section{Reference}

[1] Peter Lorimer 'On the Finite Two Dimensional Linear Groups'. Journal of Algebra (to appear).

University of Auckland

Auckland, New Zealand 